\title{
Article
}

\section{Refinements of two fractional versions of Hadamard inequalities for Caputo fractional derivatives and related results}

\author{
Ghulam Farid $^{1, *}$, Atiq Ur Rehman ${ }^{1}$, Sidra Bibi ${ }^{1}$ and Yu-Ming Chu ${ }^{2,3}$ \\ 1 Department of Mathematics, COMSATS University Islamabad, Attock Campus, Pakistan. \\ 2 Department of Mathematics, Huzhou University, Huzhou 313000, P. R. China. \\ 3 Hunan Provincial Key Laboratory of Mathematical Modeling and Analysis in Engineering, Changsha University of \\ Science \& Technology, Changsha 410114, P. R. China. \\ * Correspondence: ghlmfarid@ciit-attock.edu.pk
}

Academic Editor: Merve Ilkhan Kara

Received: 11 October 2020; Accepted: 30 December 2020; Published: 9 January 2021.

\begin{abstract}
The aim of this paper is to study the fractional Hadamard inequalities for Caputo fractional derivatives of strongly convex functions. We obtain refinements of two known fractional versions of the Hadamard inequality for convex functions. By applying identities for Caputo fractional derivatives we get refinements of error bounds of these inequalities. The given results simultaneously provide refinements as well as generalizations of already known inequalities.
\end{abstract}

Keywords: Convex fuction, strongly convex function, Hadamard inequality, Riemann-Liouville fractional integrals.

MSC: 33E12, 26A33, 26A51.

\section{Introduction}

C onvex functions are important in the theory of mathematical inequalities, many well-known results are direct implications of these functions. The concepts of various types of new convex functions are obtained from the definition of convex functions. Their generalizations, extensions and refinements proved very beneficial in mathematical analysis, mathematical statistics, optimization theory, graph theory.

The main objective of this paper is to obtain refinements of two versions of the Hadamard inequality for Caputo fractional derivatives of convex functions proved [1,2]. The refinements of their error estimations are also established. For this purpose we employ the definition of strongly convex functions. In the following we give the definitions of functions utilized for getting the new and related results.

Definition 1. [3] A function $\psi: I \rightarrow \mathbb{R}$, where $I$ is an interval in $\mathbb{R}$, is said to be convex if undermentioned inequality holds:

$$
\psi\left(x_{0} z+(1-z) y_{0}\right) \leq z \psi\left(x_{0}\right)+(1-z) \psi\left(y_{0}\right), z \in[0,1], x_{0}, y_{0} \in I .
$$

Strongly convex function was introduced by Polyak in [4].

Definition 2. Let $D$ be a convex subset of $\mathbb{X},(\mathbb{X},\|\|$.$) be a normed space. A function \psi: D \subset \mathbb{X} \rightarrow \mathbb{R}$ is called strongly convex function with modulus $C$ if it satisfies

$$
\psi\left(x_{0} z+(1-z) y_{0}\right) \leq z \psi\left(x_{0}\right)+(1-z) \psi\left(y_{0}\right)-C z(1-z)\left\|x_{0}-y_{0}\right\|^{2}
$$

$\forall x_{0}, y_{0} \in D, z \in[0,1]$ and $C>0$. If we take $C=0$ in (2) we get inequality (1).

Many authors have been inventing the properties and applications of strongly convex function for more information, [5-10]. In this paper we will use this definition for normed space $\mathbb{R}$.

A well-known inequality named the Hadamard inequality is an equivalent interpretation of convex function. It is given as follows: 
Theorem 1. Let $\psi: I \rightarrow \mathbb{R}$ be a convex function on interval $I \subset \mathbb{R}$ and $x_{0}, y_{0} \in I$ where $x_{0}<y_{0}$. Then the undermentioned inequality holds:

$$
\psi\left(\frac{x_{0}+y_{0}}{2}\right) \leq \frac{1}{y_{0}-x_{0}} \int_{x_{0}}^{y_{0}} \psi(x) d x \leq \frac{\psi\left(x_{0}\right)+\psi\left(y_{0}\right)}{2} .
$$

If order in (3) is reversed, then it holds for concave function.

In the literature of mathematical inequalities the Hadamard inequality appears in various forms for convex and related functions. In [11] it was studied for Riemann-Liouville fractional integrals and after it many researchers started to get its versions for different kinds of fractional integral operators and functions. In [1,2], we have derived such inequalities for Caputo fractional derivatives. For the fractional integral inequalities we refer readers to [11-25]. The Caputo fractional derivatives is defined as follows.

Definition 3. [26] Let $\beta>0$ and $\beta \notin\{1,2,3, \ldots\}, n=[\beta]+1, \psi \in A C^{n}\left[x_{0}, y_{0}\right]$. Then Caputo fractional derivatives of order $\beta$ are defined as:

$$
{ }^{C} D_{x_{0}+}^{\beta} \psi(x)=\frac{1}{\Gamma(n-\beta)} \int_{x_{0}}^{x} \frac{\psi^{(n)}(z)}{(x-z)^{\beta-n+1}} d z, x>x_{0}
$$

and

$$
{ }^{C} D_{y_{0}-}^{\beta} \psi(x)=\frac{(-1)^{n}}{\Gamma(n-\beta)} \int_{x}^{y_{0}} \frac{\psi^{(n)}(z)}{(z-x)^{\beta-n+1}} d z, x<y_{0}
$$

If $\beta=n \in\{1,2,3, \ldots\}$ and usual derivative of order $n$ exists, then $\left({ }^{C} D_{x_{0}+}^{\beta} \psi\right)(x)=\psi^{(n)}(x)$, whereas $\left({ }^{C} D_{y_{0}-}^{\beta} \psi\right)(x)=(-1)^{n} \psi^{(n)}(x)$. In particular we have

$$
\left({ }^{C} D_{x_{0}+}^{0} \psi\right)(x)=\left({ }^{C} D_{y_{0}-}^{0} \psi\right)(x)=\psi(x),
$$

where $n=1$ and $\beta=0$.

Next, we give the results which are directly related to the findings of this paper. Farid et al., [1] proved the undermentioned Hadamard inequality for Caputo fractional derivatives:

Theorem 2. Let $\psi:\left[x_{0}, y_{0}\right] \rightarrow \mathbb{R}$ be the function with $\psi \in C^{n}\left[x_{0}, y_{0}\right]$ and $0 \leq x_{0}<y_{0}$. Also let $\psi^{(n)}$ be positive and convex function on $\left[x_{0}, y_{0}\right]$. Then the undermentioned inequality holds for Caputo fractional derivatives:

$$
\psi^{(n)}\left(\frac{x_{0}+y_{0}}{2}\right) \leq \frac{\Gamma(n-\beta+1)}{2\left(y_{0}-x_{0}\right)^{n-\beta}}\left[\left({ }^{c} D_{x_{0}^{+}}^{\beta} \psi\right)\left(y_{0}\right)+(-1)^{n}\left({ }^{c} D_{y_{0}^{-}}^{\beta} \psi\right)\left(x_{0}\right)\right] \leq \frac{\psi^{(n)}\left(x_{0}\right)+\psi^{(n)}\left(y_{0}\right)}{2} .
$$

Lemma 1. [1] For Caputo fractional derivatives the following identity holds:

$$
\begin{aligned}
& \frac{\psi^{(n)}\left(x_{0}\right)+\psi^{(n)}\left(y_{0}\right)}{2}-\frac{\Gamma(n-\beta+1)}{2\left(y_{0}-x_{0}\right)^{n-\beta}}\left[\left({ }^{C} D_{x_{0}^{+}}^{\beta} \psi\right)\left(y_{0}\right)+(-1)^{n}\left({ }^{C} D_{y_{0}^{-}}^{\beta} \psi\right)\left(x_{0}\right)\right] \\
& =\frac{y_{0}-x_{0}}{2} \int_{0}^{1}\left[(1-z)^{n-\beta}-z^{n-\beta}\right] \psi^{(n+1)}\left(z x_{0}+(1-z) y_{0}\right) d z .
\end{aligned}
$$

Farid et al., [1] also proved the undermentioned inequality for Caputo fractional derivatives:

Theorem 3. Let $0 \leq x_{0}<y_{0}, \psi \in C^{n+1}\left[x_{0}, y_{0}\right]$ and also let $\left|\psi^{(n+1)}\right|$ be convex. Then for Caputo fractional derivatives we have:

$$
\begin{aligned}
& \left|\frac{\psi^{(n)}\left(x_{0}\right)+\psi^{(n)}\left(y_{0}\right)}{2}-\frac{\Gamma(n-\beta+1)}{2\left(y_{0}-x_{0}\right)^{n-\beta}}\left[\left({ }^{C} D_{x_{0}^{+}}^{\beta} \psi\right)\left(y_{0}\right)+(-1)^{n}\left({ }^{C} D_{y_{0}^{-}}^{\beta} \psi\right)\left(x_{0}\right)\right]\right| \\
& \leq \frac{\left(y_{0}-x_{0}\right)}{2(n-\beta+1)}\left(1-\frac{1}{2^{n-\beta}}\right)\left[\left|\psi^{(n+1)}\left(x_{0}\right)\right|+\left|\psi^{(n+1)}\left(y_{0}\right)\right|\right] .
\end{aligned}
$$


Kang et al., [2] proved the undermentioned version of the Hadamard inequality for Caputo fractional derivatives:

Theorem 4. Under assumptions of Theorem 2, for Caputo fractional derivatives we have:

$$
\begin{aligned}
\psi^{(n)}\left(\frac{x_{0}+y_{0}}{2}\right) & \leq \frac{2^{n-\beta-1} \Gamma(n-\beta+1)}{\left(y_{0}-x_{0}\right)^{n-\beta}}\left[\left({ }^{C} D_{\left(\frac{x_{0}+y_{0}}{2}\right)^{+}}^{\beta} \psi\right)\left(y_{0}\right)+(-1)^{n}\left({ }^{C} D_{\left(\frac{x_{0}+y_{0}}{2}\right)^{-}}^{\beta} \psi\right)\left(x_{0}\right)\right] \\
& \leq \frac{\psi^{(n)}\left(x_{0}\right)+\psi^{(n)}\left(y_{0}\right)}{2} .
\end{aligned}
$$

Lemma 2. [2] For Caputo fractional derivatives the following identity holds:

$$
\begin{aligned}
& \frac{2^{n-\beta-1} \Gamma(n-\beta+1)}{\left(y_{0}-x_{0}\right)^{n-\beta}}\left[\left({ }^{C} D_{\left(\frac{x_{0}+y_{0}}{2}\right)^{+}}^{\beta} \psi\right)\left(y_{0}\right)+(-1)^{n}\left({ }^{C} D_{\left(\frac{x_{0}+y_{0}}{2}\right)^{-}}^{\beta} \psi\right)\left(x_{0}\right)\right]-\psi^{(n)}\left(\frac{x_{0}+y_{0}}{2}\right) \\
& =\frac{y_{0}-x_{0}}{4}\left[\int_{0}^{1} z^{n-\beta} \psi^{(n+1)}\left(\frac{z}{2} x_{0}+\left(\frac{2-z}{2}\right) y_{0}\right)-\int_{0}^{1} z^{n-\beta} \psi^{(n+1)}\left(\frac{2-z}{2} x_{0}+\frac{z}{2} y_{0}\right) d z\right] .
\end{aligned}
$$

Kang et al., [2] also proved the undermentioned inequalities for Caputo fractional derivatives:

Theorem 5. Let $\psi:\left[x_{0}, y_{0}\right] \rightarrow \mathbb{R}$ be a differentiable mapping on $\left(x_{0}, y_{0}\right)$ with $\psi \in C^{n+1}\left[x_{0}, y_{0}\right]$ and $x_{0}<y_{0}$. If $\left|\psi^{(n+1)}\right|^{q}$ is convex on $\left[x_{0}, y_{0}\right]$ for $q \geq 1$, then for Caputo fractional derivatives we have:

$$
\begin{aligned}
& \left|\frac{2^{n-\beta-1} \Gamma(n-\beta+1)}{\left(y_{0}-x_{0}\right)^{n-\beta}}\left[\left({ }^{C} D_{\left(\frac{x_{0}+y_{0}}{2}\right)^{+}}^{\beta} \psi\right)\left(y_{0}\right)+(-1)^{n}\left({ }^{C} D_{\left(\frac{x_{0}+y_{0}}{2}\right)^{-}}^{\beta} \psi\right)\left(x_{0}\right)\right]-\psi^{(n)}\left(\frac{x_{0}+y_{0}}{2}\right)\right| \\
& \leq \frac{y_{0}-x_{0}}{4(n-\beta+1)}\left(\frac{1}{2(n-\beta+2)}\right)^{\frac{1}{q}}\left[\left((n-\beta+1)\left|\psi^{(n+1)}\left(x_{0}\right)\right|^{q}+(n-\beta+3)\left|\psi^{(n+1)}\left(y_{0}\right)\right|^{q}\right)^{\frac{1}{q}}\right. \\
& \left.\quad+\left((n-\beta+3)\left|\psi^{(n+1)}\left(x_{0}\right)\right|^{q}+(n-\beta+1)\left|\psi^{(n+1)}\left(y_{0}\right)\right|^{q}\right)^{\frac{1}{q}}\right] .
\end{aligned}
$$

Theorem 6. [2] Under assumptions of Theorem 3, for Caputo fractional derivatives we have:

$$
\begin{aligned}
& \left|\frac{2^{n-\beta-1} \Gamma(n-\beta+1)}{\left(y_{0}-x_{0}\right)^{n-\beta}}\left[\left({ }^{C} D_{\left(\frac{x_{0}+y_{0}}{2}\right)^{+}}^{\beta} \psi\right)\left(y_{0}\right)+(-1)^{n}\left({ }^{C} D_{\left(\frac{x_{0}+y_{0}-}{2}\right)^{-}}^{\beta} \psi\right)\left(x_{0}\right)\right]-\psi^{(n)}\left(\frac{x_{0}+y_{0}}{2}\right)\right| \\
& \leq \frac{y_{0}-x_{0}}{4}\left(\frac{1}{n p-\beta p+1}\right)^{\frac{1}{p}}\left[\left(\frac{\left|\psi^{(n+1)}\left(x_{0}\right)\right|^{q}+3\left|\psi^{(n+1)}\left(y_{0}\right)\right|^{q}}{4}\right)^{\frac{1}{q}}+\left(\frac{3\left|\psi^{(n+1)}\left(x_{0}\right)\right|^{q}+\left|\psi^{(n+1)}\left(y_{0}\right)\right|^{q}}{4}\right)^{\frac{1}{q}}\right] \\
& \leq \frac{y_{0}-x_{0}}{4}\left(\frac{4}{3(n p-\beta p+1)}\right)^{\frac{1}{p}}\left[\left|\psi^{(n+1)}\left(x_{0}\right)\right|+\left|\psi^{(n+1)}\left(y_{0}\right)\right|\right]
\end{aligned}
$$

where $\frac{1}{p}+\frac{1}{q}=1$.

The paper is organized in the manner that, in Section 2 we give two versions of the Hadamard inequality via Caputo fractional derivatives of strongly convex functions. These inequalities give refinements of Theorems 2 and 4. In Section 3, by employing identities stated in Lemmas 1 and 2, the refinements of Theorems 3 and 6 are presented.

\section{Main results}

The following results gives the refinement of the Hadamard inequality for Caputo fractional derivatives stated in Theorem 2.

Theorem 7. Let $\psi:\left[x_{0}, y_{0}\right] \rightarrow \mathbb{R}$ be the positive function such that $\psi \in C^{n}\left[x_{0}, y_{0}\right]$ and $0 \leq x_{0}<y_{0}$. If $\psi^{(n)}$ is strongly convex function with modulus $C$, then for Caputo fractional derivatives we have: 


$$
\begin{aligned}
& \psi^{(n)}\left(\frac{x_{0}+y_{0}}{2}\right)+\frac{C\left(y_{0}-x_{0}\right)^{2}\left[(\beta-n+2)+(n-\beta)^{2}\right]}{4(n-\beta+1)(n-\beta+2)} \\
& \leq \frac{\Gamma(n-\beta+1)}{2\left(y_{0}-x_{0}\right)^{n-\beta}}\left[\left({ }^{C} D_{x_{0}^{+}}^{\beta} \psi\right)\left(y_{0}\right)+(-1)^{n}\left({ }^{C} D_{y_{0}^{-}}^{\beta} \psi\right)\left(x_{0}\right)\right] \\
& \leq \frac{\left[\psi^{(n)}\left(x_{0}\right)+\psi^{(n)}\left(y_{0}\right)\right]}{2}-\frac{C(n-\beta)\left(y_{0}-x_{0}\right)^{2}}{(n-\beta+1)(n-\beta+2)}
\end{aligned}
$$

with $\beta>0$.

Proof. Since $\psi^{(n)}$ is strongly convex function with modulus $C$, for $x, y \in\left[x_{0}, y_{0}\right]$, we have

$$
\psi^{(n)}\left(\frac{x+y}{2}\right) \leq \frac{\psi^{(n)}(x)+\psi^{(n)}(y)}{2}-\frac{C}{4}|x-y|^{2} .
$$

Let $x=x_{0} z+(1-z) y_{0}$ and $y=y_{0} z+(1-z) x_{0}, z \in[0,1]$. Then we have

$$
2 \psi^{(n)}\left(\frac{x_{0}+y_{0}}{2}\right) \leq \psi^{(n)}\left(x_{0} z+(1-z) y_{0}\right)+\psi^{(n)}\left(y_{0} z+(1-z) x_{0}\right)-\left(\frac{C}{2}\right)(1-2 z)^{2}\left(y_{0}-x_{0}\right)^{2}
$$

Multiplying (16) with $z^{n-\beta-1}$ on both sides and making integration over $[0,1]$ we get

$$
\begin{aligned}
& 2 \psi^{(n)}\left(\frac{x_{0}+y_{0}}{2}\right) \int_{0}^{1} z^{n-\beta-1} d z \leq \int_{0}^{1} \psi^{(n)}\left(x_{0} z+(1-z) y_{0}\right) z^{n-\beta-1} d z \\
& \quad+\int_{0}^{1} \psi^{(n)}\left(y_{0} z+(1-z) x_{0}\right) z^{n-\beta-1} d z-\frac{C}{2}\left(y_{0}-x_{0}\right)^{2} \int_{0}^{1}(1-2 z)^{2} z^{n-\beta-1} d z .
\end{aligned}
$$

By using change of variables and computing the last integral, from (17) we get

$$
\begin{aligned}
\frac{2}{n-\beta} \psi^{(n)}\left(\frac{x_{0}+y_{0}}{2}\right) \leq & \frac{1}{y_{0}-x_{0}} \int_{x_{0}}^{y_{0}} \psi^{(n)}(x)\left(\frac{y_{0}-x}{y_{0}-x_{0}}\right)^{n-\beta-1} d x+\frac{1}{y_{0}-x_{0}} \int_{x_{0}}^{y_{0}} \psi^{(n)}(y)\left(\frac{y-x_{0}}{y_{0}-x_{0}}\right)^{n-\beta-1} d y \\
& -\frac{C\left(y_{0}-x_{0}\right)^{2}\left[(\beta-n+2)+(n-\beta)^{2}\right]}{2(n-\beta)(n-\beta+1)(n-\beta+2)} .
\end{aligned}
$$

Further it takes the following form

$$
\begin{aligned}
\psi^{(n)}\left(\frac{x_{0}+y_{0}}{2}\right) \leq & \frac{\Gamma(n-\beta+1)}{2\left(y_{0}-x_{0}\right)^{n-\beta}}\left[\left({ }^{C} D_{x_{0}^{+}}^{\beta} \psi\right)\left(y_{0}\right)+(-1)^{n}\left({ }^{c} D_{y_{0}^{-}}^{\beta} \psi\right)\left(x_{0}\right)\right] \\
& -\frac{C\left(y_{0}-x_{0}\right)^{2}\left[(\beta-n+2)+(n-\beta)^{2}\right]}{4(n-\beta+1)(n-\beta+2)} .
\end{aligned}
$$

Since $\psi^{(n)}$ is strongly convex function with modulus $C$, for $z \in[0,1]$, then one has

$$
\psi^{(n)}\left(z x_{0}+(1-z) y_{0}\right)+\psi^{(n)}\left(z y_{0}+(1-z) x_{0}\right) \leq \psi^{(n)}\left(x_{0}\right)+\psi^{(n)}\left(y_{0}\right)-2 C z(1-z)\left(y_{0}-x_{0}\right)^{2}
$$

Multiplying (20) with $z^{n-\beta-1}$ on both sides and making integration over $[0,1]$ we get

$$
\begin{aligned}
& \int_{0}^{1} \psi^{(n)}\left(z x_{0}+(1-z) y_{0}\right) z^{n-\beta-1} d z+\int_{0}^{1} \psi^{(n)}\left(z y_{0}+(1-z) x_{0}\right) z^{n-\beta-1} d z \\
& \leq \int_{0}^{1} \psi^{(n)}\left(x_{0}\right) z^{n-\beta-1} d z+\int_{0}^{1} \psi^{(n)}\left(y_{0}\right) z^{n-\beta-1} d z-2 C\left(y_{0}-x_{0}\right)^{2} \int_{0}^{1}(1-z) z^{n-\beta} d z .
\end{aligned}
$$

By using change of variables and computing the last integral, from (21) we get 


$$
\begin{aligned}
& \frac{1}{y_{0}-x_{0}} \int_{x_{0}}^{y_{0}} \psi^{(n)}(x)\left(\frac{y_{0}-x}{y_{0}-x_{0}}\right)^{n-\beta-1} d x+\frac{1}{y_{0}-x_{0}} \int_{x_{0}}^{y_{0}} \psi^{(n)}(y)\left(\frac{y-x_{0}}{y_{0}-x_{0}}\right)^{n-\beta-1} d y \\
& \leq \frac{\psi^{(n)}\left(x_{0}\right)+\psi^{(n)}\left(y_{0}\right)}{n-\beta}-\frac{2 C\left(y_{0}-x_{0}\right)^{2}}{(n-\beta+1)(n-\beta+2)} .
\end{aligned}
$$

Further, it takes the following form

$$
\frac{\Gamma(n-\beta+1)}{2\left(y_{0}-x_{0}\right)^{n-\beta}}\left[\left({ }^{C} D_{x_{0}^{+}}^{\beta} \psi\right)\left(y_{0}\right)+(-1)^{n}\left({ }^{C} D_{y_{0}^{-}}^{\beta} \psi\right)\left(x_{0}\right)\right] \leq \frac{\left[\psi^{(n)}\left(x_{0}\right)+\psi^{(n)}\left(y_{0}\right)\right]}{2}-\frac{C(n-\beta)\left(y_{0}-x_{0}\right)^{2}}{(n-\beta+1)(n-\beta+2)} .
$$

Inequalities (19) and (22) constituted the required inequality.

Remark 1. If $C=0$ in (14), then we will get the fractional Hadamard inequality stated in Theorem 2, otherwise its refinement is obtained.

The upcoming result is the refinement of another version of the Hadamard inequality for Caputo fractional derivatives stated in Theorem 4.

Theorem 8. Let $\psi:\left[x_{0}, y_{0}\right] \rightarrow \mathbb{R}$ be the positive function such that $\psi \in C^{n}\left[x_{0}, y_{0}\right]$ and $0 \leq x_{0}<y_{0}$. If $\psi^{(n)}$ is a strongly convex function with modulus $C$, then the undermentioned inequality for Caputo fractional derivatives holds:

$$
\begin{aligned}
& \psi^{(n)}\left(\frac{x_{0}+y_{0}}{2}\right)+\frac{C\left(y_{0}-x_{0}\right)^{2}}{2(n-\beta+1)(n-\beta+2)} \\
& \leq \frac{2^{n-\beta-1} \Gamma(n-\beta+1)}{\left(y_{0}-x_{0}\right)^{n-\beta}}\left[\left({ }^{C} D_{\left(\frac{x_{0}+y_{0}}{2}\right)^{+}}^{\beta} \psi\right)\left(y_{0}\right)+(-1)^{n}\left({ }^{C} D_{\left(\frac{x_{0}+y_{0}}{2}\right)^{-}}^{\beta} \psi\right)\left(x_{0}\right)\right] \\
& \leq \frac{\psi^{(n)}\left(x_{0}\right)+\psi^{(n)}\left(y_{0}\right)}{2}-\frac{C(n-\beta)(b-a)^{2}(n-\beta+3)}{4(n-\beta+1)(n-\beta+2)},
\end{aligned}
$$

with $\beta>0$.

Proof. Let $x=x_{0} \frac{z}{2}+y_{0}\left(\frac{2-z}{2}\right)$ and $y=x_{0}\left(\frac{2-z}{2}\right)+y_{0} \frac{z}{2}, z \in[0,1]$ in (15), then we have

$$
2 \psi^{(n)}\left(\frac{x_{0}+y_{0}}{2}\right) \leq \psi^{(n)}\left(x_{0} \frac{z}{2}+y_{0}\left(\frac{2-z}{2}\right)\right)+\psi^{(n)}\left(x_{0}\left(\frac{2-z}{2}\right)+y_{0} \frac{z}{2}\right)-\frac{C}{2}\left(y_{0}-x_{0}\right)^{2}(1-z)^{2} .
$$

Multiplying (24) with $z^{n-\beta-1}$ on both sides and making integration over $[0,1]$ we get

$$
\begin{aligned}
& 2 \psi^{(n)}\left(\frac{x_{0}+y_{0}}{2}\right) \int_{0}^{1} z^{n-\beta-1} d z \leq \int_{0}^{1} \psi^{(n)}\left(x_{0} \frac{z}{2}+y_{0}\left(\frac{2-z}{2}\right)\right) z^{n-\beta-1} d z \\
& \quad+\int_{0}^{1} \psi^{(n)}\left(x_{0}\left(\frac{2-z}{2}\right)+y_{0} \frac{z}{2}\right) z^{n-\beta-1} d z-\frac{C}{2}\left(y_{0}-x_{0}\right)^{2} \int_{0}^{1}(1-z)^{2} z^{n-\beta-1} d z
\end{aligned}
$$

By using change of variables and computing the last integral, from (25) we get

$$
\begin{aligned}
\frac{2}{n-\beta} \psi^{(n)}\left(\frac{x_{0}+y_{0}}{2}\right) \leq & \int_{\frac{x_{0}+y_{0}}{2}}^{y_{0}}\left(\frac{2\left(y_{0}-x\right)}{y_{0}-x_{0}}\right)^{n-\beta-1} \psi^{(n)}(x) \frac{2 d x}{y_{0}-x_{0}}+\int_{x_{0}}^{\frac{x_{0}+y_{0}}{2}}\left(\frac{2\left(y-x_{0}\right)}{y_{0}-x_{0}}\right)^{n-\beta-1} \\
& \times \psi^{(n)}(y) \frac{2 d y}{y_{0}-x_{0}}-\frac{C\left(y_{0}-x_{0}\right)^{2}}{(n-\beta)(n-\beta+1)(n-\beta+2)} .
\end{aligned}
$$


Further, it takes the following form

$$
\begin{aligned}
\psi^{(n)}\left(\frac{x_{0}+y_{0}}{2}\right) \leq & \frac{2^{n-\beta-1} \Gamma(n-\beta+1)}{\left(y_{0}-x_{0}\right)^{n-\beta}}\left[\left({ }^{C} D_{\left(\frac{x_{0}+y_{0}}{2}\right)^{+}}^{\beta} \psi\right)\left(y_{0}\right)+(-1)^{n}\left({ }^{C} D_{\left(\frac{x_{0}+y_{0}}{2}\right)^{-}}^{\beta} \psi\right)\left(x_{0}\right)\right] \\
& -\frac{C\left(y_{0}-x_{0}\right)^{2}}{2(n-\beta+1)(n-\beta+2)} .
\end{aligned}
$$

Since $\psi^{(n)}$ is strongly convex function and $z \in[0,1]$, we have the following inequality:

$$
\psi^{(n)}\left(x_{0} \frac{z}{2}+y_{0}\left(\frac{2-z}{2}\right)\right)+\psi^{(n)}\left(x_{0}\left(\frac{2-z}{2}\right)+y_{0} \frac{z}{2}\right) \leq \psi^{(n)}\left(x_{0}\right)+\psi^{(n)}\left(y_{0}\right)-\frac{C z(2-z)\left(y_{0}-x_{0}\right)^{2}}{2} .
$$

Multiplying (28) with $z^{n-\beta-1}$ on both sides and making integration over $[0,1]$ we get

$$
\begin{aligned}
& \int_{0}^{1} \psi^{(n)}\left(x_{0} \frac{z}{2}+y_{0}\left(\frac{2-z}{2}\right)\right) z^{n-\beta-1} d z+\int_{0}^{1} \psi^{(n)}\left(x_{0}\left(\frac{2-z}{2}\right)+y_{0} \frac{z}{2}\right) z^{n-\beta-1} d z \\
& \leq \int_{0}^{1} \psi^{(n)}\left(x_{0}\right) z^{n-\beta-1} d z+\int_{0}^{1} \psi^{(n)}\left(y_{0}\right) z^{n-\beta-1} d z-\frac{C\left(y_{0}-x_{0}\right)^{2}}{2} \int_{0}^{1}(2-z) z^{n-\beta} d z
\end{aligned}
$$

By using change of variables and computing the last integral, from (29) we get

$$
\begin{aligned}
& \frac{2}{y_{0}-x_{0}} \int_{\frac{x_{0}+y_{0}}{2}}^{y_{0}} \psi^{(n)}(x)\left(\frac{2\left(y_{0}-x\right)}{y_{0}-x_{0}}\right)^{n-\beta-1} d x+\frac{2}{y_{0}-x_{0}} \int_{x_{0}}^{\frac{x_{0}+y_{0}}{2}} \psi^{(n)}(y)\left(\frac{2\left(y-x_{0}\right)}{y_{0}-x_{0}}\right)^{n-\beta-1} d y \\
& \leq \frac{\psi^{(n)}\left(x_{0}\right)+\psi^{(n)}\left(y_{0}\right)}{n-\beta}-\frac{C\left(y_{0}-x_{0}\right)^{2}(n-\beta+3)}{2(n-\beta+1)(n-\beta+2)} .
\end{aligned}
$$

Further, it takes the following form

$$
\begin{aligned}
& \frac{2^{n-\beta-1} \Gamma(n-\beta+1)}{\left(y_{0}-x_{0}\right)^{n-\beta}}\left[\left({ }^{c} D_{\left(\frac{x_{0}+y_{0}}{2}\right)^{+}}^{\beta} \psi\right)\left(y_{0}\right)+(-1)^{n}\left({ }^{c} D_{\left(\frac{x_{0}+y_{0}}{2}\right)^{-}}^{\beta} \psi\right)\left(x_{0}\right)\right] \\
& \leq \frac{\psi^{(n)}\left(x_{0}\right)+\psi^{(n)}\left(y_{0}\right)}{2}-\frac{C(n-\beta)\left(y_{0}-x_{0}\right)^{2}(n-\beta+3)}{4(n-\beta+1)(n-\beta+2)} .
\end{aligned}
$$

From (27) and (31), (23) can be obtained.

Remark 2. If $C=0$ in (23), then we will get the fractional Hadamard inequality stated in Theorem 4, otherwise its refinement is obtained.

\section{Error bounds of fractional Hadamard inequalities}

In this section we give refinements of the error bounds of fractional Hadamard inequalities for Caputo fractional derivatives:

Theorem 9. Let $\psi:\left[x_{0}, y_{0}\right] \rightarrow \mathbb{R}$ be the function such that $\psi \in C^{n+1}\left[x_{0}, y_{0}\right]$ and $0 \leq x_{0}<y_{0}$. If $\left|\psi^{(n+1)}\right|$ is a strongly convex function on $\left[x_{0}, y_{0}\right]$, then the undermentioned inequality for Caputo fractional derivatives holds:

$$
\begin{aligned}
& \left|\frac{\psi^{(n)}\left(x_{0}\right)+\psi^{(n)}\left(y_{0}\right)}{2}-\frac{\Gamma(n-\beta+1)}{2\left(y_{0}-x_{0}\right)^{n-\beta}}\left[\left({ }^{C} D_{x_{0}^{+}}^{\beta} \psi\right)\left(y_{0}\right)+(-1)^{n}\left({ }^{C} D_{y_{0}^{-}}^{\beta} \psi\right)\left(x_{0}\right)\right]\right| \\
& \leq \frac{\left(y_{0}-x_{0}\right)}{2(n-\beta+1)}\left(1-\frac{1}{2^{n-\beta}}\right)\left[\left|\psi^{(n+1)}\left(x_{0}\right)\right|+\left|\psi^{(n+1)}\left(y_{0}\right)\right|\right]-\frac{C\left(y_{0}-x_{0}\right)^{3}}{(n-\beta+2)(n-\beta+3)}\left(1-\frac{n-\beta+4}{2^{n-\beta+2}}\right),
\end{aligned}
$$

with $\beta>0$.

Proof. By applying Lemma 1 and the strong convexity of $\left|\psi^{(n+1)}\right|$, we find 


$$
\begin{aligned}
& \left|\frac{\psi^{(n)}\left(x_{0}\right)+\psi^{(n)}\left(y_{0}\right)}{2}-\frac{\Gamma(n-\beta+1)}{2\left(y_{0}-x_{0}\right)^{n-\beta}}\left[\left({ }^{C} D_{x_{0}^{+}}^{\beta} \psi\right)\left(y_{0}\right)+(-1)^{n}\left({ }^{C} D_{y_{0}^{-}}^{\beta} \psi\right)\left(x_{0}\right)\right]\right| \\
& \leq \frac{y_{0}-x_{0}}{2} \int_{0}^{1}\left|(1-z)^{n-\beta}-z^{n-\beta}\right|\left|\psi^{(n+1)}\left(z x_{0}+(1-z) y_{0}\right)\right| d z \\
& \leq \frac{y_{0}-x_{0}}{2}\left[\int_{0}^{1}\left|(1-z)^{n-\beta}-z^{n-\beta}\right|\left(z\left|\psi^{(n+1)}\left(x_{0}\right)\right|+(1-z)\left|\psi^{(n+1)}\left(y_{0}\right)\right|-C z(1-z)\left|y_{0}-x_{0}\right|^{2}\right) d z\right] \\
& \leq \frac{y_{0}-x_{0}}{2}\left[\int_{0}^{1 / 2}\left((1-z)^{n-\beta}-z^{n-\beta}\right)\left(z\left|\psi^{(n+1)}\left(x_{0}\right)\right|+(1-z)\left|\psi^{(n+1)}\left(y_{0}\right)\right|-C z(1-z)\left|y_{0}-x_{0}\right|^{2}\right) d z\right. \\
& \left.\quad+\int_{1 / 2}^{1}\left(z^{n-\beta}-(1-z)^{n-\beta}\right)\left(z\left|\psi^{(n+1)}\left(x_{0}\right)\right|+(1-z)\left|\psi^{(n+1)}\left(y_{0}\right)\right|-C z(1-z)\left|y_{0}-x_{0}\right|^{2}\right) d z\right] .
\end{aligned}
$$

In the following we compute integrals appearing on the right side of inequality (33):

$$
\begin{aligned}
\int_{0}^{1 / 2}\left((1-z)^{n-\beta}-z^{n-\beta}\right)\left(z\left|\psi^{(n+1)}\left(x_{0}\right)\right|+(1-z)\left|\psi^{(n+1)}\left(y_{0}\right)\right|-C z(1-z)\left|y_{0}-x_{0}\right|^{2}\right) d z \\
=\left|\psi^{(n+1)}\left(x_{0}\right)\right| \int_{0}^{1 / 2}\left(z(1-z)^{n-\beta}-z^{n-\beta+1}\right) d z+\left|\psi^{(n+1)}\left(y_{0}\right)\right| \int_{0}^{1}\left((1-z)^{n-\beta+1}-(1-z) z^{n-\beta}\right) d z \\
\quad-C\left(y_{0}-x_{0}\right)^{2}\left(\int_{0}^{1 / 2} z(1-z)^{n-\beta+1} d z-\int_{0}^{1 / 2} z^{n-\beta+1}(1-z) d z\right) \\
=\left|\psi^{(n+1)}\left(x_{0}\right)\right|\left(\frac{1}{(n-\beta+1)(n-\beta+2)}-\frac{(1 / 2)^{n-\beta+1}}{n-\beta+1}\right)+\left|\psi^{(n+1)}\left(y_{0}\right)\right| \\
\quad \times\left(\frac{1}{(n-\beta+2)}-\frac{(1 / 2)^{n-\beta+1}}{n-\beta+1}\right)-\frac{C\left(y_{0}-x_{0}\right)^{2}}{(n-\beta+2)(n-\beta+3)}\left[1-\frac{(n-\beta+4)}{2^{n-\beta+2}}\right], \\
\int_{1 / 2}^{1}\left(z^{n-\beta}-(1-z)^{n-\beta}\right)\left(z\left|\psi^{(n+1)}\left(x_{0}\right)\right|+(1-z)\left|\psi^{(n+1)}\left(y_{0}\right)\right|-C z(1-z)\left|y_{0}-x_{0}\right|^{2}\right) d z \\
=\left|\psi^{(n+1)}\left(x_{0}\right)\right| \int_{1 / 2}^{1}\left(z^{n-\beta+1}-z(1-z)^{n-\beta}\right) d z+\left|\psi^{(n+1)}\left(y_{0}\right)\right| \int_{1}^{1}\left((1-z) z^{n-\beta}-(1-z)^{n-\beta+1}\right) d z \\
-C\left(y_{0}-x_{0}\right)^{2}\left(\int_{1 / 2}^{1}(1-z) z^{n-\beta+1} d z-\int_{1 / 2}^{1} z(1-z)^{n-\beta+1} d z\right) \\
=\left|\psi^{(n+1)}\left(x_{0}\right)\right|\left(\frac{1}{n-\beta+2}-\frac{(1 / 2)^{n-\beta+1}}{n-\beta+1}\right)+\left|\psi^{(n+1)}\left(y_{0}\right)\right| \\
\quad \times\left(\frac{(1 / 2)^{n-\beta+1}}{(n-\beta+1)(n-\beta+2)}\right)-\frac{C\left(y_{0}-x_{0}\right)^{2}}{n-\beta+1}\left[1-\frac{n-\beta+4}{2^{n-\beta+2}}\right] .
\end{aligned}
$$

By putting the values from (34) and (35) in (33), we get (32).

Remark 3. If $C=0$ in (32), we get the fractional Hadamard inequality which is stated in Theorem 3 , otherwise its refinement is obtained.

By using Lemma 2, we give the following error bounds of Caputo fractional derivative inequality (23).

Theorem 10. Let $\psi:\left[x_{0}, y_{0}\right] \rightarrow \mathbb{R}$ be the function such that $\psi \in C^{n+1}\left[x_{0}, y_{0}\right]$ and $0 \leq x_{0}<y_{0}$. If $\left|\psi^{(n+1)}\right|^{q}$ is strongly convex function on $\left[x_{0}, y_{0}\right]$ for $q \geq 1$, then the undermentioned inequality for Caputo fractional derivatives holds:

$$
\begin{aligned}
& \left|\frac{2^{n-\beta-1} \Gamma(n-\beta+1)}{\left(y_{0}-x_{0}\right)^{n-\beta}}\left[\left({ }^{C} D_{\left(\frac{x_{0}+y_{0}}{2}\right)^{+}}^{\beta} \psi\right)\left(y_{0}\right)+(-1)^{n}\left({ }^{C} D_{\left(\frac{x_{0}+y_{0}}{2}\right)^{-}}^{\beta} \psi\right)\left(x_{0}\right)\right]-\psi^{(n)}\left(\frac{x_{0}+y_{0}}{2}\right)\right| \\
& \leq \frac{y_{0}-x_{0}}{4(n-\beta+1)}\left(\frac{1}{2(n-\beta+2)}\right)^{\frac{1}{q}}\left[\left((n-\beta+1)\left|\psi^{(n+1)}\left(x_{0}\right)\right|^{q}+(n-\beta+3)\left|\psi^{(n+1)}\left(y_{0}\right)\right|^{q}-\frac{C\left(y_{0}-x_{0}\right)^{2}(n-\beta+1)(n-\beta+4)}{2(n-\beta+3)}\right)^{\frac{1}{q}}\right. \\
& \left.\quad+\left((n-\beta+3)\left|\psi^{(n+1)}\left(x_{0}\right)\right|^{q}+(\beta+1)\left|\psi^{(n+1)}\left(y_{0}\right)\right|^{q}-\frac{C\left(y_{0}-x_{0}\right)^{2}(n-\beta+1)(n-\beta+4)}{2(n-\beta+3)}\right)^{\frac{1}{q}}\right]
\end{aligned}
$$


Proof. By applying Lemma 2 using power mean inequality, we have

$$
\begin{aligned}
& \left|\frac{2^{n-\beta-1} \Gamma(n-\beta+1)}{\left(y_{0}-x_{0}\right)^{n-\beta}}\left[\left({ }^{C} D_{\left(\frac{x_{0}+y_{0}}{2}\right)^{+}}^{\beta} \psi\right)\left(y_{0}\right)+(-1)^{n}\left({ }^{C} D_{\left(\frac{x_{0}+y_{0}}{2}\right)^{-}}^{\beta} \psi\right)\left(x_{0}\right)\right]-\psi^{(n)}\left(\frac{x_{0}+y_{0}}{2}\right)\right| \\
& \leq \frac{y_{0}-x_{0}}{4}\left[\int_{0}^{1} z^{n-\beta}\left|\psi^{(n+1)}\left(x_{0} \frac{z}{2}+y_{0}\left(\frac{2-z}{2}\right)\right)\right| d z+\int_{0}^{1} z^{n-\beta}\left|\psi^{(n+1)}\left(x_{0}\left(\frac{2-z}{2}\right)+y_{0} \frac{z}{2}\right)\right| d z\right] \\
& \leq \frac{y_{0}-x_{0}}{4}\left(\int_{0}^{1} z^{n-\beta} d z\right)^{1-\frac{1}{q}}\left[\left(\int_{0}^{1} z^{n-\beta}\left|\psi^{(n+1)}\left(x_{0} \frac{z}{2}+y_{0}\left(\frac{2-z}{2}\right)\right)\right|^{q} d z\right)^{\frac{1}{q}}\right. \\
& \left.\quad+\left(\int_{0}^{1} z^{n-\beta} \mid \psi^{(n+1)}\left(x_{0}\left(\frac{2-z}{2}\right)+y_{0} \frac{z}{2}\right)^{q} d z\right)^{\frac{1}{q}}\right],
\end{aligned}
$$

Now, applying strong convexity of $\left|\psi^{(n+1)}\right|$, we have

$$
\begin{aligned}
\leq & \frac{y_{0}-x_{0}}{4(n-\beta+1)^{\frac{1}{p}}}\left[\left(\left|\psi^{(n+1)}\left(x_{0}\right)\right|^{q} \int_{0}^{1} \frac{z^{n-\beta+1}}{2} d z+\left|\psi^{(n+1)}\left(y_{0}\right)\right|^{q} \int_{0}^{1} \frac{2 z^{n-\beta}-z^{n-\beta+1}}{2} d z\right.\right. \\
& \left.-\frac{C\left(y_{0}-x_{0}\right)^{2}}{4} \int_{0}^{1}\left(2 z^{n-\beta+1}-z^{n-\beta+2}\right) d z\right)^{\frac{1}{q}}+\left(\left|\psi^{(n+1)}\left(x_{0}\right)\right|^{q} \int_{0}^{1} \frac{2 z^{n-\beta}-z^{n-\beta+1}}{2} d z\right. \\
& \left.\left.+\left|\psi^{(n+1)}\left(y_{0}\right)\right|^{q} \int_{0}^{1} \frac{z^{n-\beta+1}}{2} d z-\frac{C\left(y_{0}-x_{0}\right)^{2}}{4} \int_{0}^{1}\left(2 z^{n-\beta+1}-z^{n-\beta+2}\right) d z\right)^{\frac{1}{q}}\right] \\
= & \frac{y_{0}-x_{0}}{4(n-\beta+1)^{\frac{1}{p}}}\left(\frac{1}{2(n-\beta+1)(n-\beta+2)}\right)^{\frac{1}{q}}\left[\left((n-\beta+1)\left|\psi^{(n+1)}\left(x_{0}\right)\right|^{q}\right.\right. \\
& \left.+(n-\beta+3)\left|\psi^{(n+1)}\left(y_{0}\right)\right|^{q}-\frac{C\left(y_{0}-x_{0}\right)^{2}(n-\beta+1)(n-\beta+4)}{2(n-\beta+3)}\right)^{\frac{1}{q}} \\
& \left.+\left((n-\beta+3)\left|\psi^{(n+1)}\left(x_{0}\right)\right|^{q}+(n-\beta+1)\left|\psi^{(n+1)}\left(y_{0}\right)\right|^{q}-\frac{C\left(y_{0}-x_{0}\right)^{2}(n-\beta+1)(n-\beta+4)}{2(n-\beta+3)}\right)^{\frac{1}{q}}\right]
\end{aligned}
$$

inequality (36) is obtained.

Remark 4. If $C=0$ in (36), then we will get inequality stated in Theorem 5, otherwise its refinement is obtained.

Theorem 11. Let $\psi:\left[x_{0}, y_{0}\right] \rightarrow \mathbb{R}$ be a differentiable mapping on $\left(x_{0}, y_{0}\right)$ with $\psi \in C^{n+1}\left[x_{0}, y_{0}\right]$ and $x_{0}<y_{0}$. If $\left|\psi^{(n+1)}\right|^{q}$ is a strongly convex function on $\left[x_{0}, y_{0}\right]$ for $q>1$, then the undermentioned inequality for Caputo fractional derivatives holds:

$$
\begin{aligned}
& \left|\frac{2^{n-\beta-1} \Gamma(n-\beta+1)}{\left(y_{0}-x_{0}\right)^{n-\beta}}\left[\left({ }^{C} D_{\left(\frac{x_{0}+y_{0}}{2}\right)^{+}}^{\beta} \psi\right)\left(y_{0}\right)+(-1)^{n}\left({ }^{C} D_{\left(\frac{x_{0}+y_{0}}{2}\right)^{-}}^{\beta} \psi\right)\left(x_{0}\right)\right]-\psi^{(n)}\left(\frac{x_{0}+y_{0}}{2}\right)\right| \\
& \leq \frac{y_{0}-x_{0}}{16}\left(\frac{4}{n p-\beta p+1}\right)^{\frac{1}{p}}\left[\left(\left(\left|\psi^{(n+1)}\left(x_{0}\right)\right|+3^{\frac{1}{q}}\left|\psi^{(n+1)}\left(y_{0}\right)\right|\right)^{q}-\frac{2 C\left(y_{0}-x_{0}\right)^{2}}{3}\right)^{\frac{1}{q}}\right. \\
& \left.\quad+\left(\left(3^{\frac{1}{q}}\left|\psi^{(n+1)}\left(x_{0}\right)\right|+\left|\psi^{(n+1)}\left(y_{0}\right)\right|\right)^{q}-\frac{2 C\left(y_{0}-x_{0}\right)^{2}}{3}\right)^{\frac{1}{q}}\right] .
\end{aligned}
$$

Proof. By applying Lemma 2 and with the help of modulus property, we get

$$
\begin{aligned}
& \left|\frac{2^{n-\beta-1} \Gamma(n-\beta+1)}{\left(y_{0}-x_{0}\right)^{n-\beta}}\left[\left({ }^{C} D_{\left(\frac{x_{0}+y_{0}}{2}\right)^{+}}^{\beta} \psi\right)\left(y_{0}\right)+(-1)^{n}\left({ }^{C} D_{\left(\frac{x_{0}+y_{0}}{2}\right)^{-}}^{\beta} \psi\right)\left(x_{0}\right)\right]-\psi^{(n)}\left(\frac{x_{0}+y_{0}}{2}\right)\right| \\
& \leq \frac{y_{0}-x_{0}}{4}\left[\int_{0}^{1} z^{n-\beta}\left|\psi^{(n+1)}\left(\frac{z}{2} x_{0}+y_{0} \frac{(2-z)}{2}\right)\right| d z+\int_{0}^{1} z^{n-\beta}\left|\psi^{(n+1)}\left(\frac{2-z}{2} x_{0}+\frac{z}{2} y_{0}\right)\right| d z\right] .
\end{aligned}
$$


Now applying Hölder's inequality, we get

$$
\begin{aligned}
& \left|\frac{2^{n-\beta-1} \Gamma(n-\beta+1)}{\left(y_{0}-x_{0}\right)^{n-\beta}}\left[\left({ }^{C} D_{\left(\frac{x_{0}+y_{0}}{2}\right)^{+}}^{\beta} \psi\right)\left(y_{0}\right)+(-1)^{n}\left({ }^{C} D_{\left(\frac{x_{0}+y_{0}}{2}\right)^{-}}^{\beta} \psi\right)\left(x_{0}\right)\right]-\psi^{(n)}\left(\frac{x_{0}+y_{0}}{2}\right)\right| \\
& \leq \frac{y_{0}-x_{0}}{4}\left(\frac{1}{n p-\beta p+1}\right)^{\frac{1}{p}}\left[\left(\int_{0}^{1}\left|\psi^{(n+1)}\left(\frac{z}{2} x_{0}+y_{0} \frac{(2-z)}{2}\right)\right| d z\right)^{\frac{1}{q}}\right. \\
& \left.\quad+\left(\int_{0}^{1} z^{n-\alpha}\left|\psi^{(n+1)}\left(\frac{2-z}{2} x_{0}+\frac{z}{2} y_{0}\right)\right| d z\right)^{\frac{1}{q}}\right] .
\end{aligned}
$$

Using strong convexity of $\left|\psi^{(n+1)}\right| q$, we get

$$
\begin{aligned}
&\left|\frac{2^{n-\beta-1} \Gamma(n-\beta+1)}{\left(y_{0}-x_{0}\right)^{n-\beta}}\left[\left({ }^{C} D_{\left(\frac{x_{0}+y_{0}}{2}\right)^{+}}^{\beta} \psi\right)\left(y_{0}\right)+(-1)^{n}\left({ }^{C} D_{\left(\frac{x_{0}+y_{0}}{2}\right)^{-}}^{\beta} \psi\right)\left(x_{0}\right)\right]-\psi^{(n)}\left(\frac{x_{0}+y_{0}}{2}\right)\right| \\
& \leq \frac{y_{0}-x_{0}}{4}\left(\frac{1}{n p-\beta p+1}\right)^{\frac{1}{p}}\left[\left(\left|\psi^{(n+1)}\left(x_{0}\right)\right|^{q} \int_{0}^{1} \frac{z}{2} d z+\left|\psi^{(n+1)}\left(y_{0}\right)\right|^{q} \int_{0}^{1}\left(\frac{2-z}{2}\right) d z\right.\right. \\
&\left.-\frac{C\left(y_{0}-x_{0}\right)^{2}}{4} \int_{0}^{1}\left(2 z-z^{2}\right) d z\right)^{\frac{1}{q}}+\left(\left|\psi^{(n+1)}\left(x_{0}\right)\right|^{q} \int_{0}^{1}\left(\frac{2-z}{2}\right) d z\right. \\
&\left.\left.+\left|\psi^{(n+1)}\left(y_{0}\right)\right|^{q} \int_{0}^{1} \frac{z}{2} d z-\frac{C\left(y_{0}-x_{0}\right)^{2}}{4} \int_{0}^{1}\left(2 z-z^{2}\right) d z\right)^{\frac{1}{q}}\right] \\
&= \frac{y_{0}-x_{0}}{16}\left(\frac{4}{n p-\beta p+1}\right)^{\frac{1}{p}}\left[\left(\left|\psi^{(n+1)}\left(x_{0}\right)\right|^{q}+3\left|\psi^{(n+1)}\left(y_{0}\right)\right|^{q}-\frac{2 C\left(y_{0}-x_{0}\right)^{2}}{3}\right)^{\frac{1}{q}}\right. \\
&\left.+\left(3\left|\psi^{(n+1)}\left(x_{0}\right)\right|^{q}+\left|\psi^{(n+1)}\left(y_{0}\right)\right|^{q}-\frac{2 C\left(y_{0}-x_{0}\right)^{2}}{3}\right)^{\frac{1}{q}}\right] \\
& \leq \frac{y_{0}-x_{0}}{16}\left(\frac{4}{n p-\beta p+1}\right)^{\frac{1}{p}}\left[\left(\left(\left|\psi^{(n+1)}\left(x_{0}\right)\right|+3^{\frac{1}{q}}\left|\psi^{(n+1)}\left(y_{0}\right)\right|\right)^{q}-\frac{2 C\left(y_{0}-x_{0}\right)^{2}}{3}\right)^{\frac{1}{q}}\right. \\
&\left.+\left(\left(3^{\frac{1}{q}}\left|\psi^{(n+1)}\left(x_{0}\right)\right|+\left|\psi^{(n+1)}\left(y_{0}\right)\right|\right)^{q}-\frac{2 C\left(y_{0}-x_{0}\right)^{2}}{3}\right)^{\frac{1}{q}}\right] .
\end{aligned}
$$

Here we have used the fact that $\left(a_{1}+b_{1}\right)^{q} \geq\left(a_{1}\right)^{q}+\left(b_{1}\right)^{q}$, where $q>1, a_{1}, b_{1} \geq 0$. This completes the proof.

Remark 5. If $C=0$ in (37), then we will get inequality stated in Theorem 6, otherwise its refinement is obtained.

\section{Concluding Remarks}

This paper provides refinements of fractional versions of the Hadamard inequalities for Caputo fractional derivatives using strongly convex functions. Also, refinements of error bounds of the Hadamard inequalities are given by using well established identities. Further, the authors are working for refinements of other well known fractional integral inequalities by employing strongly convexities of other kinds.

Acknowledgments: The research was supported by the National Natural Science Foundation of China (Grant Nos. $11971142,11871202,61673169,11701176,11626101,11601485)$. The research work of Atiq Ur Rehman and Ghulam Farid is supported by the Higher Education Commission of Pakistan with Project No. 7962 and Project No. 5421 respectively.

Author Contributions: All authors contributed equally to the writing of this paper. All authors read and approved the final manuscript.

Conflicts of Interest: "The authors declare no conflict of interest."

\section{References}


[1] Farid, G., Naqvi, S., \& Javed, A. (2017). Hadamard and Fejér-Hadamard inequalities and related results via Caputo fractional derivatives. Bulletin of Mathematical Analysis and Applications, 9(3), 16-30.

[2] Kang, S. M., Farid, G., Nazeer, W., \& Naqvi, S. (2019). A version of the Hadamard inequality for Caputo fractional derivatives and related results. Journal of Computational Analysis \& Applications, 26(1), 962-972.

[3] Roberts, A. W. (1993). Convex Functions. In Handbook of Convex Geometry (pp. 1081-1104). North-Holland.

[4] Polyak, B. T. (1966). Existence theorems and convergence of minimizing sequences in extremum problems with restrictions. Soviet Mathematics Doklady, 7, 72-75.

[5] Khan, M. A., Alam, F., \& Ullah, S. Z. (2019). Majorization type inequalities for strongly convex functions. Turkish Journal of Inequalities, 3(2), 62-78.

[6] Makò, J., \& Hézy, A. (2016). On strongly convex functions. Carpathian Journal of Mathematics, 32(1), 87-95.

[7] Merentes, N., \& Nikodem, K. (2010). Remarks on strongly convex functions. Aequationes Mathematicae, 80(1-2), 193-199.

[8] Nikodem, K. (2014). On strongly convex functions and related classes of functions. In Handbook of Functional Equations (pp. 365-405). Springer, New York, NY.

[9] Nikodem, K., \& Páles, Z. (2011). Characterizations of inner product spaces by strongly convex functions. Banach Journal of Mathematical Analysis, 5(1), 83-87.

[10] Vial, J. P. (1982). Strong convexity of sets and functions. Journal of Mathematical Economics, 9(1-2), 187-205.

[11] Sarikaya, M. Z., Set, E., Yaldiz, H., \& Basak, N. (2013). Hermite-Hadamard's inequalities for fractional integrals and related fractional inequalities. Mathematical and Computer Modelling, 57(9-10), 2403-2407.

[12] Agarwal, P. (2017). Some inequalities involving Hadamard-type $k$-fractional integral operators. Mathematical Methods in the Applied Sciences, 40(11), 3882-3891.

[13] Agarwal, P., Jleli, M., \& Tomar, M. (2017). Certain Hermite-Hadamard type inequalities via generalized $k$-fractional integrals. Journal of Inequalities and Applications, 2017, Artilce No. 55.

[14] Chen, F. (2014). On Hermite-Hadamard type inequalities for Riemann-Liouville fractional integrals via two kinds of convexity. Chinese Journal of Mathematics, 2014, Article ID 173293.

[15] Ekinci, A., \& Özdemir, M. E. (2019). Some new integral inequalities via Riemann-Liouville integral operators. Applied and Computational Mathematics, 18(3), 288-295.

[16] Farid, G., Javed, A., \& Rehman, A. U. (2017). On Hadamard inequalities for $n$-times differentiable functions which are relative convex via Caputo $k$-fractional derivatives. Nonlinear Analysis Forum, 22(2), 17-28.

[17] Farid, G., Javed, A., ur Rehman, A., \& Qureshi, M. I. (2017). On Hadamard-type inequalities for differentiable functions via Caputo $k$-fractional derivatives. Cogent Mathematics \& Statistics, 4(1), 1355429.

[18] Farid, G., Rehman, A. U., \& Zahra, M. (2016). On Hadamard inequalities for $k$-fractional integrals. Nonlinear Functional Analysis and Applications, 21(3), 463-478.

[19] Guo, S., Chu, Y. M., Farid, G., Mehmood, S., \& Nazeer, W. (2020). Fractional Hadamard and Fejér-Hadamard inequalities associated with exponentially $(s, m)$-convex functions. Journal Function Spaces, 2020, Article ID 2410385.

[20] Hong, G., Farid, G., Nazeer, W., Akbar, S. B., Pécarić, J., Zou, Z., \& Geng, S. (2020). Boundedness of fractional integral operators containing Mittag-Leffler function via exponentially s-convex functions. Journal of Mathematics, 2020, Article ID 3584105.

[21] Kang, S. M., Farid, G., Nazeer, W., \& Naqvi, S. (2019). A version of the Hadamard inequality for Caputo fractional derivatives and related results. Journal of Computational Analysis E Applications, 27(6), 962-972.

[22] Kunt, M., \& İscan, İ. (2018). Fractional Hermite-Hadamard-Fejér type inequalities for GA-convex functions. Turkish Journal of Inequalities, 2(1), 1-20.

[23] Khan, M. A., \& Khan, T. U. (2017). Parameterized Hermite-Hadamard type inequalities for fractional integrals. Turkish Journal of Inequalities, 1(1), 26-37.

[24] Qi, H. X., Yussouf, M., Mehmood, S., Chu, Y. M., \& Farid, G. (2020). Fractional integral versions of Hermite-Hadamard type inequality for generalized exponentially convexity. AIMS Mathematics, 5(6), 6030-6042.

[25] Set, E., Choi, J., \& Celik, B. (2018). Certain Hermite-Hadamard type inequalities involving generalized fractional integral operators. Revista de la Real Academia de Ciencias Exactas, Físicas y Naturales. Serie A. Matemáticas, 112(4), 1539-1547.

[26] Kilbas, A. A., Srivastava, H. M., \& Trujillo, J. J. (2006). Theory and Applications of Fractional Differential Equations (Vol. 204). Elsevier.

(C) 2021 by the authors; licensee PSRP, Lahore, Pakistan. This article is an open access article distributed under the terms and conditions of the Creative Commons Attribution (CC-BY) license (http://creativecommons.org/licenses/by/4.0/). 